This is the author's final, peer-reviewed manuscript as accepted for publication. The publisher-formatted version may be available through the publisher's web site or your institution's library.

\title{
Transcriptomics of induced defense responses to greenbug aphid feeding in near isogenic wheat lines.
}

Srirama Krishna Reddy, Yiqun Weng, Jackie C. Rudd, Alina Akhunova, Shuyu Liu

\section{How to cite this manuscript}

If you make reference to this version of the manuscript, use the following information:

Reddy, S. K., Rudd, J. C., Akhunova, A., \& Liu, S. (2013). Transcriptomics of induced defense responses to greenbug aphid feeding in near isogenic wheat lines. Retrieved from http://krex.ksu.edu

\section{Published Version Information}

Citation: Reddy, S. K., Rudd, J. C., Akhunova, A., \& Liu, S. (2013). Transcriptomics of induced defense responses to greenbug aphid feeding in near isogenic wheat lines. Plant Science, 212, 26-36.

Copyright: @ 2013 Elsevier Ireland Ltd.

Digital Object Identifier (DOI):10.1016/j.plantsci.2013.08.002

Publisher's Link: http://dx.doi.org/10.1016/j.plantsci.2013.08.002

This item was retrieved from the K-State Research Exchange (K-REx), the institutional repository of Kansas State University. K-REx is available at http://krex.ksu.edu 


\section{Transcriptomics of induced defense responses to greenbug aphid feeding in near isogenic wheat lines}

Srirama Krishna Reddy ${ }^{a}$, Yiqun Weng ${ }^{a}, *, \#$, Jackie C. Rudd ${ }^{a}$, Alina Akhunova ${ }^{b}$, Shuyu Liu ${ }^{a}$ *

${ }^{a}$ Texas A\&M AgriLife Research, Texas A\&M University, Amarillo, TX, United States

${ }^{\mathrm{b}}$ Integrated Genomics Facility, Kansas State University, 4024 Throckmorton, Manhattan, KS, United States

* Corresponding Authors:

Y. Weng: Vegetable Crops Research Unit, USDA-ARS, Horticulture Department, University of Wisconsin, Madison, WI 53706, USA. Phone: 001-608- 262-0028.

S. Liu: Texas A\&M AgriLife Research, Texas A\&M University, 6500 Amarillo Blvd W., Amarillo, TX 79106, USA. Phone: 001-806-677-5600; Fax: 001-806-677-5644.

E-mail addresses: skrishnareddy@ag.tamu.edu (S. Krishna Reddy), weng4@wisc.edu or yiqun.weng@ars.usda.gov (Y. Weng), JCRudd@ag.tamu.edu (J.C. Rudd), Akhunova@kstate.edu (A. Akhunova), SLiu@ag.tamu.edu (S. Liu).

\# Present address: USDA-ARS Vegetable Crops Research Unit, Horticulture Department, University of Wisconsin, Madison, WI, United States. ${ }^{1}$

${ }^{1}$ Abbreviations: JA - Jasmonic acid; ET - Ethylene; ABA - Abscisic acid; SA - Salicylic acid; GA - Gibberellic acid; BR - Brassinosteroid; R - resistant; S - Susceptible; RB - Resistant Bulk; SB - Susceptible Bulk; hai - Hours After Inoculation; FC - Fold Change. 


\begin{abstract}
The greenbug aphid, Schizaphis graminum (Rondani) is an important cereal pest, periodically threatening wheat yields in the United States and around the world. The single dominant gene, Gb3-based resistance is highly durable against prevailing greenbug biotypes under field conditions; however, the molecular mechanisms of $G b 3$-mediated defense responses remain unknown. We used Affymetrix GeneChip Wheat Genome Arrays to investigate the transcriptomics of host defense responses upon greenbug feeding on resistant and susceptible bulks (RB and SB, respectively) derived from two near-isogenic lines. The study identified 692 differentially expressed transcripts and further functional classification recognized 122 transcripts that are putatively associated to mediate biotic stress responses. In RB, Gb3-mediated resistance resulted in activation of transmembrane receptor kinases and signaling-related transcripts involved in early signal transduction cascades. While in SB, transcripts mediating final steps in jasmonic acid biosynthesis, redox homeostasis, peroxidases, glutathione Stransferases, and notable defense-related secondary metabolites were induced. Also transcripts involved in callose and cell wall decomposition were elevated SB, plausibly to facilitate uninterrupted feeding operations. These results suggest that $G b 3$-mediated resistance is less vulnerable to cell wall modification and the data provides ample tools for further investigations concerning $R$ gene based model of resistance.
\end{abstract}

Key Words - Aphid resistance; Greenbug; jasmonic acid; microarray; transcriptomics; wheat 


\section{Introduction}

Bread wheat (Triticum aestivum) is the third largest cereal crop in the world (http://faostat.fao.org/) and second most important cereal in the United States with a production of 60 million tons during 2010. Most of the hard-red winter wheat in the US is cultivated in the Southern Great Plains where the yields are hampered by several phloem feeding insect pests primarily, the greenbug aphid, Schizaphis graminum (Rondani). Economic losses due to greenbug vary each year but are estimated to reach $\$ 405$ million annually (http://www.wheatworld.org/wp-content/uploads/Wheat-Pest-Initiative-FY11-Final.pdf). Given the number of alternative hosts, biotypes, modes of reproduction, and frequency of outbreaks, the greenbug poses a threat to farming and continues to vex the scientific community. Deployment of resistance cultivars is an important component of integrated pest management for control of greenbug aphids, but host resistance can potentially be defeated by new virulent biotypes [1]. Hence, a thorough understanding of the physiological and molecular basis of resistance mechanisms serves as a key to the development of cultivars with durable resistance and better tactics for insect control.

During the course of evolution, plants have developed sophisticated sensory mechanisms enabling them to perceive the nature of herbivore feeding habits and to elicit appropriate defense responses. In the context of crop production, ecology, and host plant resistance, induced defense signaling plays a very important role by allowing plants to make necessary adaptation to herbivore attack [2]. The induced defense responses of greenbug aphids with piercing/sucking feeding behavior (preferentially feeding on phloem sap) contrast to those of chewing insects. The phloem-feeders pierce through the physical barrier and consume photosynthates to alter photosynthate composition and resource allocation that is primarily driven for defense $[3,4]$. For successful feeding operations, aphids navigate their stylets between intercellular spaces to reach phloem sieve elements [5]. Once the connection is established with phloem sieve elements, the aphids may feed continuously for hours to days and even weeks. To facilitate an uninterrupted feeding the aphids secrete salivary substances which may not only assist in easy stylet penetration but also serve as an elicitor to trigger downstream pathways and suppress the plant induced defense cues $[6,7]$. 
Perception of herbivore induced elicitors and effectors by the membrane bound receptors activate the putative herbivore or damage-associated molecular patterns (HAMP or DAMP), some that are parallel to pathogen or microbe-associated molecular patterns (PAMP or MAMP), critical in basal or induced immune system [8-10]. Since most phloem-feeders cause minimal physical damage to the plant tissue, the resulting defense responses are similar to those associated with PAMP or MAMP and are recognized by pattern recognition receptors (PRRs) present on the surface of plant cells [3, 8]. Perception of phloem-feeding herbivores by $R$ genes similar to disease resistance $\mathrm{R}$ proteins encodes a coiled coil nucleotide-binding leucine rich repeat (CC-NBS-LRR) protein [11-14]. Perception of feeding behavior by plants with $R$ gene was shown to activate phytohormone, salicylic acid (SA)-dependent signaling cascade and callose deposition [14]. The detailed signaling cascades involving NB-LRR motif has been thoroughly summarized in the context of disease resistance $[15,16]$ and plant-aphid interactions [2] suggesting that the induced defense responses involve multiple signal transduction pathways.

Following perception of piercing/sucking feeding patterns, plants activate early signal networks those trigger massive transcriptional reprogramming and downstream responses to defend against phloem-feeders [17]. The early signal transduction events induced by phloemfeeding insects are largely mediated by calcium flux, reactive oxygen species (ROS), phytohormones, volatiles organic compounds, and nonvolatile secondary metabolites that can serve as repellants, toxins, and even attract natural enemies [9, 18-20]. Calcium ions $\left(\mathrm{Ca}^{2+}\right)$ in the plants serve as secondary messengers mediating developmental responses, stress signaling, and herbivore attack [18]. After sensing aphid feeding, $\mathrm{Ca}^{2+}$ sensors activate downstream defense signaling cascades by increasing expression of calmodulin, calmodulin binding proteins, and calcium-dependent protein kinases (CDPKs) [21]. The role of ROS in mediating herbivore attack by either chewing or piercing/sucking insects is unquestionable; however, the nature of response depends on type of herbivory and duration and intensity of feeding [4, 22-24].

The phytohormones SA, jasmonic acid (JA), and ethylene (ET) activate herbivore induced signals via independent, antagonistic, and synergistic pathways and interface with other hormones auxin, abscisic acid (ABA), brassinosteroid (BR), gibberellins (GA), and cytokinin (CK) [25-28]. Additionally, as part of the defense mechanisms against phloem-feeding insects and other herbivores, plants are known to alter secondary metabolites, glutathione S-transferases 
(GSTs), peroxidases, and redox homeostasis [19, 29-31]. As a defensive mechanism, plants resistant to phloem feeding herbivores increase callose deposition in sieve tubes; while susceptible plants promote callose-decomposing enzymes such as $\beta$-1,3-glucanase (also present in aphid saliva), resulting in unplugging of phloem occlusion [32].

From a plant breeding perspective, $R$ gene-mediated host defenses play critical roles against herbivore damage; however, the detailed physiological and molecular basis of gene-for-gene interactions in the grass genomes like wheat remains unclear $[33,34]$. Host resistance to piercing/sucking insects is usually controlled by single or major genes [35]. Our previous studies indicated that host plant resistance to greenbug infestation in the wheat cultivar TAM 110 is due to a single dominant gene $G b 3$, which has been mapped in the distal end of wheat chromosome arm 7DL and tagged with molecular markers [36-38]. Previous behavioral and phenotypic studies on greenbug biotype E infestation in the resistant (TXGBE273) and susceptible (TXGBE281) NILs of Gb3 suggested that antixenosis, antibiosis, and tolerance were responsible for host plant resistance against the greenbug aphid [39-43]. Using these preconditioned R and S NILs we found that Gb3-mediated induced defense responses were systemic, rendering uninfected young leaves more protected [43]. Systemic induced resistance was also noticed in S NIL but at a much lower level compared to Gb3-induced resistance in R NIL. When feeding on resistant TXGBE273 plants, the greenbugs spent more time wandering on the leaf surface compared to susceptible plants (TAM 105) where feeding begins soon after infestation [41]. However, the molecular basis of Gb3-mediated early defense responses and associated signal transduction pathways triggered by greenbug feeding remain unknown.

The current study was conducted to explore the global transcriptomic responses of greenbug feeding to elucidate the molecular mechanisms underlying $G b 3$-mediated as well as basal defense responses. We used Affymetrix GeneChip Wheat Genome Arrays to assess the transcriptomic changes in the resistant and susceptible bulks (RB and SB, respectively) within 24 and $48 \mathrm{~h}$ after greenbug infestation. A functional classification was performed based on pairwise biologically meaningful comparisons constructed between $\mathrm{R}$ and $\mathrm{S}$ genotypes at 0,24 , and $48 \mathrm{~h}$ after greenbug infestation and the results were validated using qRT-PCR.

\section{Materials and methods}




\subsection{Plant materials, growth conditions, and greenbug infestation}

$\mathrm{F}_{8}$ recombinant inbred lines (RILs) derived from two near isogenic lines of the greenbug resistance gene $G b 3$, TXGBE273 (Gb3Gb3) and susceptible TXGBE281 ( $g b 3 g b 3)$ [37, 44] were developed. Sixteen RILs, eight homozygous resistant (Gb3Gb3) and eight homozygous recessive, were chosen to construct two bulks, the resistant bulk (RB) and susceptible bulk (SB), as the starting materials for transcriptome profiling in the present study. Homozygosity of the 16 RILs at the $G b 3$ locus was verified in three consecutive generations $\left(\mathrm{F}_{6}, \mathrm{~F}_{7}\right.$, and $\left.\mathrm{F}_{8}\right)$ in greenbug biotype E infestation tests with at least 100 plants for each RIL. Nine plants from each of the 16 RILs were grown in three replicates in plastic trays using LC1 growth medium (three plants per replication per RIL). The plants were grown under controlled environmental conditions in a growth chamber with mixed fluorescent and incandescent lights providing $\sim 300 \mu \mathrm{mol} \mathrm{m}^{-2} \mathrm{~s}^{-1}$ PPFD for a $12 \mathrm{~h}$ photoperiod. Healthy plant growth conditions were maintained throughout the experiment with periodic watering. When the plants reached three-leaf stage, each plant was infested with 25 biotype E greenbugs, as previously described [42, 43].

\subsection{Sample collection and RNA preparation}

Leaf tissues from three plants of each RIL were collected at 0,24, and 48 hours after infestation (hai). Prior to leaf sample collection, all greenbug aphids were carefully removed from the seedlings with a fine hair brush. The leaf tissues were flash frozen in liquid nitrogen and stored at $-80^{\circ} \mathrm{C}$ until further processing. For RNA extraction, the leaf samples were ground into fine powder in liquid nitrogen using mortar and pestle. The total RNA was extracted using the TRIzol reagent according to manufacturer's instructions (Invitrogen, Carlsbad, CA, USA) and quantified. Then the $\mathrm{R}$ and $\mathrm{S}$ bulks were constructed in such a way that for each replication at each time point, equal amounts of total RNA from each of the eight resistant and susceptible RILs was pooled to make the RB and SB respectively. Thus 18 total RNA samples ( 2 bulks; 3 time points; and 3 replications) were prepared for subsequent expression profiling using Affymetrix GeneChip Arrays. RNA quality and concentration was determined using NanoDrop spectrophotometer and 2100 Bioanalyzer (Agilent Technologies, Santa Clara, CA, USA). All the RNA samples were of high quality and hence used for microarray analysis without any elimination. 


\subsection{Microarray assay and data analysis}

The Affymetrix GeneChip Wheat Genome Arrays (Affymetrix, Santa Clara, CA, USA) were used for transcriptomic analysis. Each wheat GeneChip array contained 61,127 probe sets representing 55,052 transcripts constructed using ESTs distributed across wheat genome. cDNA synthesis, labeling, and hybridization of the arrays were performed according to Affymetrix protocol at the Integrated Genomic Facility, Kansas State University, Manhattan, Kansas, USA. Following hybridization, chips were washed, stained and scanned using GeneChip scanner to generate the CEL and CHP files.

For data analysis, the CEL files were imported into GeneSpring software v.11.5 (Agilent Technologies, Santa Clara, CA, USA). Normalization of expression intensities on 61,290 probe sets was carried out using Robust Multichip Average (RMA) summarization algorithm [45, 46]. Quality control on samples was assessed using Principal Component Analysis (PCA) as per the Affymetrix GeneSpring GX guidelines. The PCA plots indicated that three biological replicates were located close to each other and correlation coefficients were high for replicated samples with similar treatment effects indicating less genetic background noise. The variability in the normalized expression values was corrected by filtering the probe sets based on error (retaining genes with Coefficient of Variation < 50\%) across conditions. Followed by, a two-way analysis of variance (ANOVA, $\mathrm{p}<0.01$ ) and multiple testing correction, Benjamini Hochberg approach with false discovery rate (FDR, $\mathrm{q}<0.01)$ was used to identify transcripts with statistically significant expression differences. Statistical analysis suggested that there were robust expression differences for main effects of genotype and greenbug feeding duration with significant interaction effects. Based on objectives of the study, we pursued 692 transcripts with significant expression difference showing interaction effects between genotype and greenbug feeding for detailed functional analysis. The complete list of these differentially expressed transcripts can be accessed in the supplementary data. Also, the metadata for this experiment and related array data have been submitted to the National Center for Biotechnology Information - Gene Expression Omnibus database repository (GSE45995).

Before functional analysis, nine biologically meaningful pairwise comparisons were created on the differentially expressed genes. Three pairwise comparisons between the genotypes, RB 
and SB at 0, 24, and 48 hai (R0 vs. S0, R24 vs. S24, and R48 vs. S48) and three each within the RB (R0 vs. R24, R0 vs. R48, and R24 vs. R48) and SB (S0 vs. S24, S0 vs. S48, and S24 vs. S48) were generated. The first three pairwise comparisons between the genotypes at different temporal greenbug treatment duration were selected for detailed functional analysis and are presented in the manuscript.

\subsection{MapMan analysis}

Functional classification of the differentially regulated transcripts $[47,48]$ was performed using the MapMan software version 3.5.1 (http://mapman.gabipd.org/web/guest/mapman). The transcripts imported by the ImageAnnotator module were assigned to 32 out of 36 parent BINs (hierarchical categories) defined in the MapMan software (Fig. 1). Each parent BIN was structured like a tree with sub-BINs corresponding to different biological functions and known cellular and metabolic processes. The functional BINs (or functional classes) were created for processes including but not limited to photosynthesis, redox, cell wall regulation, carbohydrate, lipid, amino acid, hormone and secondary metabolism, transport, signaling etc. The biotic stress pathway overview identifies the transcripts that are known to be part of cascade of defense signals in response to pest or pathogen attack [49] and groups into separate BINs and sub-BINs (hierarchical functional classifications). The transcripts grouped together under a major BIN (ex. signaling, cell wall, secondary metabolites, hormones, etc.) were investigated further to elucidate the defense mechanisms.

\section{5. $q R T-P C R$ analysis}

The Affymetrix based gene expression levels were validated by quantitative real-time polymerase chain reaction (qRT-PCR) using the SYBR Green detection method. The qRT-PCR analysis was performed on a Bio-Rad iCycler iQ real time PCR detection system (Bio-Rad, USA). Subset of RNA sample was drawn from the original RNA pool used for microarray to run qRT-PCR analysis. For the validation process, 16 genes of interest selected from the pool of statistically significant genes, including two reference genes with lowest standard deviation. Changes in gene expression were determined for treatments 0,24 , and 48 hai in both RB and SB. Primers were designed on the basis of 25mer Affymetrix probes using Beacon Designer Software (PRIMIER Biosoft, Palo Alto, CA, USA). The gene specific primers were verified on 
the agarose gel and then SuperScript II reverse transcriptase (Invitrogen, Catalog \# 18064-014) and T7 poly (T) primer was used to generate cDNA. Primer efficiency tests performed on all the primers using serial dilution of one of the cDNA samples showed efficiency from 95 to $100 \%$. Four genes showing multiple peaks in the melting curve analysis were eliminated from further analysis. The primers that passed the efficiency test and had a single peak in the melting curve were used for the gene expression analysis. The primer combinations and annotation information about these genes are presented in the supplemental data. The target threshold cycles were determined and changes in expression levels for a given comparison were subtracted from the counterpart. The relative expression of genes of interest in relation to a reference gene was calculated using Livak method [50].

\section{Results and discussion}

In our continued efforts to understand the molecular basis of $G b 3$-mediated defense responses, here, we present the transcriptomic investigations of greenbug aphid and wheat interactions. The current microarray study was piloted by developing bulked RILs from a cross between two NILs of Gb3 (resistant TXGBE273 and susceptible TXGBE281) to reduce the genetic background noise in the expression profiling data. The statistical analysis showed significant interaction effects between the main factors, genotype, and greenbug treatment and identified 692 differentially expressed transcripts. The global gene expression changes in response to genotypic variation and temporal greenbug treatment were better captured by the interaction effects hence, the transcripts classified under the interaction group were used for further downstream analysis and functional classification.

\subsection{Overview of greenbug induced transcriptomic changes}

Differentially expressed transcripts were imported into the MapMan software for classification based on the hierarchical functional categories, BINs and sub-BINs defined in the software [47]. Three pairwise comparisons between RB and SB (out of nine biologically meaningful comparisons) were used in functional classification to understand the global changes in response to greenbug feeding. The overview analysis in the MapMan software grouped 692 differentially expressed transcripts into 32 BINs (Fig. 1). The BIN \#35 featuring 316 transcripts not assigned to any functional class was not considered for analysis and discussion and BINs 
\#12, 24, and 32 had no genes assigned. This global overview suggested that about $88 \%$ of 376 genes grouped into MapMan BINs were classified under 15 major functional classes with 9 or more genes (Fig. 1). The BIN 26 representing 'Misc' group comprised most transcripts (58) with genes related to oxidases, peroxidase, GST, cytochrome P450, etc. The transcripts identified to mediate amino acid metabolism (39), protein related (39), transport (38), stress (24) and signaling related transcripts (23) comprised other major groups. Also, the BINs related to lipid metabolism (15), secondary metabolism (15), hormone metabolism (14), RNA (14), redox regulation (12), nucleotide metabolism (11), cell (11), development (10), and cell wall modification (9) had substantial number of genes. Other important functional categories include fermentation (8), photosynthesis (6) and photosynthetic byproduct-related processes such as oxidative pentose phosphate (OPP) cycle (6), mitochondrial electron transport (4), and tricarboxylic acid (TCA) cycle (5), etc. suggest alterations in photosynthate composition and concentration.

To understand the functional significance of induced defense responses to greenbug, we utilized the built-in biotic stress overview in MapMan software which classifies genes putatively involved in mediating biotic stress (pest or pathogen) responses [49]. The biotic stress response visualization presents substantial alteration in the transcriptome of both $\mathrm{R}$ and $\mathrm{S}$ genotypes within $24 \mathrm{~h}$ after greenbug infestation (Fig. 2). The pairwise comparison between RB and SB before greenbug infestation showed negligible differences between the genotypes (Fig. 2A); however, profound transcriptomic changes were noticed in RB compared to SB at 24 and 48 hai (Figs. 2B and 2C). These results suggest that greenbug feeding was instrumental in altered induced defense responses in RB and SB at 24 and 48 hai. A total of 122 transcripts belonging to signaling, hormone metabolism, secondary metabolites, cell wall modification, and changes in redox state, peroxidases, glutathione S-transferase (GST), beta glucanases, transcription factors, proteolysis, PR-protein related categories were found to be altered within 24 and $48 \mathrm{~h}$ of greenbug feeding (Fig. 3). The transcripts grouped in the above mentioned categories were studied in detail to understand the functional significance and the resistance mechanisms involved among R \& S genotypes in response to greenbug feeding. The annotation details and expression intensities of these differentially expressed transcripts mapped under biotic stress pathway are presented in supplemental data. 


\subsection{Perception and early signaling}

Plant recognition of greenbug presence and feeding is the first committed step in activating downstream signaling cascades to induce defense responses. Perception of invasion by phloem feeding insects has been associated with unique transmembrane CC-NB-LRR protein kinases plausibly involved in gene-for-gene mode of resistance [14]. Fourteen transcripts with more than two FC and putatively associated with perception and signal transduction pathways were altered at 24 and 48 hai in RB vs. SB comparison (Fig. 4A). Three transcripts encoding LRR family of receptor kinases proteins were found to be gradually up-regulated with the onset of greenbug infestation in RB compared to SB. The LRR transmembrane protein kinases have been implicated to trigger plant defense responses to pathogen and herbivore attack [10,51]; however, it is not clear if these transcripts along with taaffx.4501.1.a1_at, a LRR XII are involved in mediating similar responses to herbivore attack. Through a map-based cloning strategy, we recently cloned a candidate gene for $G b 3$ that putatively encodes a NB-ARC-LRR type $\mathrm{R}$ protein; however, this candidate gene was not present in the Affymetrix array (P. Azhaguvel and Y. Weng, unpublished data).

The core early signaling events in response to phloem feeding insects include changes in protein phosphorylation, calcium fluxes, ROS, phytohormones and related transcriptional regulation. Transcripts associated with calcium signaling (ta.6853.1.a1_at, a calmodulin-binding protein), sugar signaling (taaffx.813.1.s1_at), and phosphoinositide phospholipase (taaffx.117121.1.s1_at) were also altered within 24 and 48 hai (Fig. 4A). In RB, calcium and phospholipase signaling transcripts were down regulated at $0 \mathrm{~h}$, but were gradually up-regulated 24 and 48 hai. Sugar signaling was down-regulated in RB compared to SB at both 24 and 48 hai. Cysteine-rich Receptor Kinases (CRKs) belonging to DUF26 class proteins are known to mediate biotic and abiotic stress responses by activating $\mathrm{ABA}, \mathrm{SA}$, and JA hormone signaling $[52,53]$. Six transcripts related to Domain of Unknown Function 26 (DUF26) RLKs were also altered in a comparison between RB and SB after greenbug infestation (Fig. 4A). The receptor kinases including LRR and CRKs are shown to activate downstream signals by inducing production of ROS [52].

\subsection{Phytohormone signaling and transcriptional regulation}


Phytohormones play key roles in mediating herbivore induced defense responses by activating key early signal transduction pathways. Our study identified JA, ET, ABA, and auxin related transcripts that are putatively involved in mediating greenbug induced biotic stress responses (Fig. 4B). Transcripts similar to aldehyde oxidase 1 (AAOl) and ethylene forming enzyme-like (EFE-like) dioxygenase were down-regulated in RB compared to SB at both 24 and 48 hai. AAO1 is involved in the final steps of the auxin and ABA biosynthesis pathway [54] and EFE-like catalyzes ACC oxidase in the final step of ET biosynthesis [55]. Also, transcripts encoding ethylene response element binding protein 1 (EREBP1) and OsWRKY45, an ABA inducible gene with a disease resistance component [56] were altered in response greenbug feeding. Down-regulation of EREBP1, a member of AP2/ERF B-2 subfamily transcription factor in RB suggests up-regulation in SB in response to greenbug feeding is consistent with previous observations [57].

In plants, herbivore attack leads to rapid accumulation of JA; the process occurs in two organelles, chloroplast and peroxisomes. Initial steps in JA biosynthesis, generating 12-oxophytodienoic acid (OPDA) from a-linolenic acid (a-LeA) is mediated by lipoxygenase ( $L O X)$ genes in chloroplast $[58,59]$. The transitional steps involving release of OPDA from walls of plastids and uptake by peroxisomes remain obscure while the later steps in JA biosynthesis are catalyzed by OPDA reductase (OPR) and are localized to peroxisomes [58, 59]. Our study identified nine transcripts mediating JA biosynthetic process (Figs. 4B and 5). Six transcripts similar to $L O X$ genes were up-regulated in RB compared to SB within 24 and 48 hai, while three other transcripts putatively associated with OPR were down-regulated in RB compared to SB at 24 and 48 hai (Fig. 4B and 5). One transcript (ta.23763.1.s1_at) highly similar to LOX1 (rice) and LOX5 (Arabidopsis) was up-regulated 41 and 93 fold at 24 and 48 hai, respectively, in RB and was also confirmed using qRT-PCR (Fig. 7). Up-regulation of $L O X$ related transcripts suggest elevated OPDA levels in RB, while greater expression of $O P R$ related transcripts in SB propose increased JA or related oxylipins (Fig. 5). An increase in OPDA concentration in response to wounding has been documented before [60-63], while JA levels were not necessarily altered $[64,65]$. On the other hand, increased expression of $O P R$ related JA biosynthesis genes in SB could be explained by our previous observations that greenbugs begin to feed on the $\mathrm{S}$ genotype soon after infestation [41], triggering basal defense responses. 


\subsection{Downstream signaling and induced defense responses}

Perception of piercing and sucking cues could cause rapid changes in downstream signaling including production defense compounds including GSTs, peroxidases, and secondary metabolites, resulting in cell wall modification, nutritional status, and resource allocation etc.

\subsubsection{GSTs, peroxidases, and redox state related transcripts}

The GSTs in plants have antioxidant properties that can scavenge ROS and other toxic compounds, maintain redox homeostasis, and mediate biosynthesis of certain defense molecules; however, specific function vary by the class [66-68]. Our study identified altered expression of seven transcripts putatively associated with GSTs in response to greenbug infestation (Fig. 6A). Most of these transcripts were down-regulated by 2-6 folds in RB compared to SB at 24 and 48 hai. Reduced expression of GSTs in RB (or elevated expression in SB) indicated that the SB was fed by the greenbug more than RB and activated the GST-mediated defense signaling cascade. GSTF2 related transcripts localized in the plasma membrane encoding phi class of plant-specific proteins mediating phytohormone induced oxidative stress $[67,68]$. The only GST transcript upregulated in RB (ta.1878.3.s1_at) was putatively associated with membrane associated proteins in eicosanoid and glutathione metabolism (MAPEG-like protein) and is also involved in detoxification functions [69].

Like GSTs, peroxidases are also involved in defenses against pathogens and herbivores, scavenging $\mathrm{H}_{2} \mathrm{O}_{2}$, lignifying cell walls, catalyzing auxin degradation detoxification, wound healing, etc. [70]. The responses of peroxidase related transcripts showed similar trends where the majority of the transcripts were down-regulated in RB compared to SB at both 24 and 48 hai (Fig. 6B). Elevated expression of peroxidase related genes in SB also suggests that greenbug feeding had induced detoxification-related defense responses.

Ascorbate (or Vitamin C), glutathione, dismutases, and catalases are shown to inactivate ROS and help in maintaining redox homeostasis in plants $[4,71]$. Seven transcripts putatively associated to regulate redox reactions were found to be down-regulated in RB compared to SB within 24 and 48 hai (Fig. 6C). Transcript associated with oxidoreductase family enzyme, monodehydroascorbate reductase (MDAR) involved in ascorbate metabolism [72] was up- 
regulated in SB. Two transcripts similar to GDP-D-mannose 3,5-epimerase 1 (GME1) involved in ascorbate biosynthetic pathway were induced in SB at both 24 and 48 hai (Fig. 6C) suggesting increased ascorbate content for ROS detoxification [73]. Also, up-regulation of two transcripts encoding to glutathione synthase 2 (GHS2) involved in glutathione biosynthesis [74] in SB consistent with other antioxidant related transcripts suggest severe feeding-induced stress responses. Elevated expression of antioxidant related transcripts meditating biosynthesis of GSTs, peroxidases, and redox homeostasis in absence of $G b 3$ in SB suggests basal defense mechanisms have resulted in the production of $\mathrm{H}_{2} \mathrm{O}_{2}$ and other toxic compounds in response to greenbug feeding.

\subsubsection{Secondary metabolites and other defense related transcripts}

Chemical defenses in the form of secondary metabolites play a decisive role in induced defense mechanisms against pathogens and herbivore infestation [75, 76] especially phloem feeding insects [19]. Secondary metabolites of mevalonic acid pathway resulting in production of volatiles such as methyl jasmonate (MeJA), methyl salicylate (MeSA), and terpenes are induced in response to wounding or herbivore attack [19]. Greenbug infestation resulted in differential regulation of ten secondary metabolite related transcripts ( $>2 \mathrm{FC}$ ) that are putatively associated with biotic stress responses (Fig. 6D). This is in agreement with other results suggesting that greenbug feeding in S genotype caused more damage and activated transcripts related to early defenses and antioxidants. Most of these transcripts were down-regulated in RB compared to SB within 24 and 48 hai. Four transcripts (ta.6247.1.a1_x_at, ta.2925.1.s1_x_at, taaffx.86828.1.s1_x_at, and ta.9592.1.s1_at) involved in biosynthesis of mevalonic acid, a precursor in the biosynthetic pathway of four key hormones CK, ABA, GA and BR were downregulated in $\mathrm{R}$ compared to $\mathrm{S}$ within 24 hai. Two transcripts identified in phenylpropanoid and lignin biosynthesis pathway (ta.1465.1.s1_at and taaffx.108005.1.s1_at) were also downregulated in $\mathrm{RB}$ compared to SB at 24 and 48 hai. Additionally, transcripts related to tyrosine decarboxylase (ta.27284.1.s1_at), aldehyde dehydrogenase (ta.2907.1.s1_at) and cyanate hydratase (taaffx.132143.1.s1_s_at) were down regulated in RB vs. SB comparison at 24 and 48 hai while a transcript related to cinnamoyl-CoA reductase (ta.5657.1.s1_x_at) was the only one up-regulated at 24 and 48 hai (Fig. 6D). Following herbivore attack, plants face a major dilemma about resource partitioning; whether to continue to produce primary metabolites for 
growth and development or increase secondary metabolites to strengthen defense responses. Recent advances in plant-insect interaction using 'omic' methods have pressed the need for a comprehensive approach addressing both primary and secondary metabolites $[4,77]$.

\subsubsection{Cell wall modification}

When greenbugs pierce the cell wall into the leaf tissue for feeding on phloem fluids, they secrete saliva into plant cells triggering modifications in cell wall composition and structure. Studies have reported that plants up-regulate callose synthase, $\beta$-1,3-glucanase, and polygalacturonase in response to herbivore attack, which are also found in insect saliva [23, 32]. Our results identified altered expression of three transcripts associated with $\beta$-1,3-glucan hydrolases and six other transcripts associated with cell wall modification process in response to greenbug feeding (Fig. 6E). All $\beta$-glucanase related transcripts were down-regulated within 24 and 48 hai in RB vs. SB comparisons. Infestation by brown plant hopper (Nilaparvata lugens $\mathrm{Stal}$; $\mathrm{BPH}$ ), a phloem feeder in rice resulted in increased callose deposition at the site of stylet penetration in sieve tubes coupled with up-regulation of transcripts encoding $\beta$-1,3-glucanases to unplug sieve tube occlusion [32]. The expression patterns of $\beta$-1,3-glucanase genes in BPH susceptible plants following infestation was much higher compared to plants carrying two resistant genes, one of them is a potential $R$ gene $[14,32]$. Our results agree with these findings where SB showed increased expression of three $\beta$-1,3-glucanase related transcripts at 24 and 48 hai (Fig. 6E).

Among other cell wall modifying transcripts, a cellulose synthase related transcript (taaffx.107485.1.s1_at), was down-regulated at R0 vs. S0 comparison, while up-regulated by 6 and 10 fold at 24 and 48 hai comparisons, respectively. Two other transcripts encoding cellulose synthase (ta.7564.1.a1_at and ta.4084.1.s1_at) were down-regulated in RB compared to SB (Fig. $6 \mathrm{E})$. Previous transcriptomic studies have reported that cellulose synthase and other cell-wall modification genes were altered after sensing phloem feeding herbivores [65, 78-80] and hormones JA and SA [81]. Our data identified three cell wall degradation related transcripts that are putatively associated with polygalacturonase (pectinases) altered within 24 and 48 hai (Fig. $6 E)$. Pectin-degrading family members including polygalacturonases have been reported to be involved in triggering plant defenses to wounding [82]. Increased expression of transcripts 
related to $\beta$-1,3-glucanase, cellulose synthase, and pectinases in SB suggests that greenbug feeding induced cell wall remodeling to facilitate stylet penetration and successful feeding contrasting to RB.

\section{5. qRT-PCR analysis of differentially expressed genes}

To validate the changes observed in the microarray data, qRT-PCR analysis was performed on a selection of representative transcripts showing significant expression difference under both main effects and interaction effects. The pairwise relative expression differences used in the manuscript to elucidate the induced defense responses at 24 and 48 hai comparing RB and SB was considered for analysis. The transcripts used as reference genes, similar to elongation factor 1-alpha, showed similar expression difference in both microarray and qRT-PCR (Fig. 7). Notably, the transcripts with smaller relative expression difference ( $<10$ fold) between $\mathrm{RB}$ and SB at 0 (Fig. 7A), 24 (Fig. 7C), and 48 hai (Fig. 7E) showed better agreement between qRT-PCR and microarray analysis compared to transcripts showing greater relative expression difference (> 90 fold) for similar comparisons (Figs. 7B, 7D, and 7F, respectively). Annotation information about the transcripts and primer combinations used in the qRT-PCR are presented in supplementary data; functional roles of transcripts mediating JA biosynthetic pathway (LOX5) has been discussed above. Overall, the FC differences between RB and SB across time points obtained using microarray and qRT-PCR approaches were mostly consistent in capturing changes in gene expression induced by greenbug feeding.

\section{Summary}

We present herein comprehensive transcriptomic profiling of induced defense responses to greenbug feeding in winter wheat using resistant and susceptible bulks. When fed upon by greenbugs, the Gb3-mediated defenses in RB responded by activating transcripts encoding membrane-bound receptor kinases, calcium signaling, and other early biotic stress signaling pathways. In $\mathrm{SB}$, the basal herbivory defense responses induced expression of transcripts mediating biosynthesis of key phytohormones and antioxidant-related transcripts such as GSTs, peroxidase, ascorbate, and glutathione. It is likely that basal defense signaling (non GB3mediated) in SB lacks strong defense pathways to prevent accumulation of toxic compounds and subsequent cell/tissue damage and eventual plant death. Activation of more secondary 
metabolite-related transcripts along with other defense related transcripts in the SB also suggests excessive feeding and changes in photosynthate composition and allocation. Notably, elevated levels of pectinases mediating cell wall decomposition and $\beta$-1,3-glucanases that induce callose decomposition resulting in unplugging phloem occlusion suggest preferential feeding in SB compared to RB.

Together, these results suggest that in RB Gb3-mediated defense pathways prevented greenbug feeding by being less vulnerable to cell wall modification and subsequent cell damage. In comparison, non Gb3-mediated pathways in SB, the greenbugs could favorably alter cell walls, facilitating uninterrupted feeding which resulted in activation of transcripts mediating production of defense-related antioxidants and secondary metabolites. These results offer clues about Gb3-based induced defense responses in winter wheat and provide ample of resources for further investigation of $R$ gene based gene-for-gene resistance model concerning herbivore damage.

\section{COMPETING INTERESTS}

The authors declare that they have no competing interests.

\section{AUTHOR CONTRIBUTIONS}

Srirama Krishna Reddy and Yiqun Weng contributed equally to this work. YW and JCR designed the study. YW conducted all experiments prior to array hybridization including growing plants, aphid infestation, construction of resistant and susceptible bulks, and total RNA extraction. AA performed microarray and qRT-PCR experiments. SKR analyzed microarray data and prepared the manuscript under the supervision of SL and with the help of YW. All authors participated in the manuscript preparation and read and approved the final submission.

\section{ACKNOWLEDGEMENTS}

Authors acknowledge Drs. Jerry Michels and Scott Finlayson of Texas A\&M AgriLife Research and Dr. Paxton Payton of USDA-ARS, Lubbock for their critical reading and valuable inputs. The authors also thank Dr. Jianfa Bai of College of Veterinary Medicine and Nanyan Lu of Bioinformatics Center at Kansas State University for helpful discussion in experimental design 
and array data acquisition and Mandar Deshpande of K-State Integrated Genomics Facility for technical assistance. The funding for this research was contributed by Texas A\&M AgriLife Research and Texas Wheat Producers Board to S.L. and USDA-NRI grant (CSREES\# 200635301-16892) to Y.W.

\section{REFERENCES}

[1] J.D. Burd, D.R. Porter, Biotypic diversity in greenbug (Hemiptera : Aphididae): characterizing new virulence and host associations, J. Econ. Entomol., 99 (2006) 959-965. [2] F.L. Goggin, Plant-aphid interactions: molecular and ecological perspectives, Current Opinion in Plant Biology, 10 (2007) 399-408.

[3] L.L. Walling, The myriad plant responses to herbivores, J Plant Growth Regul, 19 (2000) 195-216.

[4] P.I. Kerchev, B. Fenton, C.H. Foyer, R.D. Hancock, Plant responses to insect herbivory: interactions between photosynthesis, reactive oxygen species and hormonal signalling pathways, Plant Cell Environ, 35 (2012) 441-453.

[5] W.F. Tjallingii, Salivary secretions by aphids interacting with proteins of phloem wound responses, J Exp Bot, 57 (2006) 739-745.

[6] P.W. Miles, Aphid saliva, Biol Rev Camb Philos Soc, 74 (1999) 41-85.

[7] S.A. Hogenhout, J.I.B. Bos, Effector proteins that modulate plant-insect interactions, Curr Opin Plant Biol, 14 (2011) 422-428.

[8] M. Erb, S. Meldau, G.A. Howe, Role of phytohormones in insect-specific plant reactions, Trends Plant Sci, 17 (2012) 250-259.

[9] J.Q. Wu, I.T. Baldwin, New insights into plant responses to the attack from insect herbivores, in: A. Campbell, M. Lichten, G. Schupbach (Eds.) Annu Rev Genet, Vol 44, 2010, pp. 1-24.

[10] A.F. Bent, D. Mackey, Elicitors, effectors, and R genes: The new paradigm and a lifetime supply of questions, in: Annual Review of Phytopathology, 2007, pp. 399-436.

[11] S.B. Milligan, J. Bodeau, J. Yaghoobi, I. Kaloshian, P. Zabel, V.M. Williamson, The root knot nematode resistance gene Mi from tomato is a member of the leucine zipper, nucleotide binding, leucine-rich repeat family of plant genes, Plant Cell, 10 (1998) 1307-1319.

[12] M. Rossi, F.L. Goggin, S.B. Milligan, I. Kaloshian, D.E. Ullman, V.M. Williamson, The nematode resistance gene $M i$ of tomato confers resistance against the potato aphid, Proc Natl Acad Sci USA, 95 (1998) 9750-9754.

[13] J. Klingler, R. Creasy, L.L. Gao, R.M. Nair, A.S. Calix, H.S. Jacob, O.R. Edwards, K.B. Singh, Aphid resistance in Medicago truncatula involves antixenosis and phloem-specific, inducible antibiosis, and maps to a single locus flanked by NBS-LRR resistance gene analogs, Plant Physiology, 137 (2005) 1445-1455.

[14] B. Du, W.L. Zhang, B.F. Liu, J. Hu, Z. Wei, Z.Y. Shi, R.F. He, L.L. Zhu, R.Z. Chen, B. Han, G.C. He, Identification and characterization of Bph14, a gene conferring resistance to brown planthopper in rice, Proc Natl Acad Sci USA, 106 (2009) 22163-22168.

[15] T.K. Eitas, J.L. Dangl, NB-LRR proteins: pairs, pieces, perception, partners, and pathways, Current Opinion in Plant Biology, 13 (2010) 472-477.

[16] J.M. Elmore, Z.J.D. Lin, G. Coaker, Plant NB-LRR signaling: upstreams and downstreams, Current Opinion in Plant Biology, 14 (2011) 365-371. 
[17] G.A. Thompson, F.L. Goggin, Transcriptomics and functional genomics of plant defence induction by phloem-feeding insects, J Exp Bot, 57 (2006) 755-766.

[18] M.E. Maffei, A. Mithofer, W. Boland, Insects feeding on plants: rapid signals and responses preceding the induction of phytochemical release, Phytochemistry, 68 (2007) 2946-2959.

[19] I. Kaloshian, L.L. Walling, Hemipterans as plant pathogens, in: Ann Rev Phytopathol, 2005, pp. 491-521.

[20] A. Mithofer, W. Boland, Plant defense against herbivores: chemical aspects, in: S.S. Merchant (Ed.) Annu Rev Plant Biol, Vol 63, 2012, pp. 431-450.

[21] D. Lecourieux, R. Raneva, A. Pugin, Calcium in plant defence-signalling pathways, New Phytologist, 171 (2006) 249-269.

[22] A. Kusnierczyk, P. Winge, T.S. Jorstad, J. Troczynska, J.T. Rossiter, A.M. Bones, Towards global understanding of plant defence against aphids - timing and dynamics of early Arabidopsis defence responses to cabbage aphid (Brevicoryne brassicae) attack, Plant Cell Environ, 31 (2008) 1097-1115.

[23] L.A. Kempema, X.P. Cui, F.M. Holzer, L.L. Walling, Arabidopsis transcriptome changes in response to phloem-feeding silverleaf whitefly nymphs. Similarities and distinctions in responses to aphids, Plant Physiol, 143 (2007) 849-865.

[24] M.J. Moloi, A.J. van der Westhuizen, The reactive oxygen species are involved in resistance responses of wheat to the Russian wheat aphid, J Plant Physiol, 163 (2006) 1118-1125.

[25] A. Koornneef, C.M.J. Pieterse, Cross talk in defense signaling, Plant Physiol, 146 (2008) 839-844.

[26] R. Bari, J. Jones, Role of plant hormones in plant defence responses, Plant Mol Biol, 69 (2009) 473-488.

[27] A. Ankala, R.Y. Kelley, D.E. Rowe, W.P. Williams, D.S. Luthe, Foliar herbivory triggers local and long distance defense responses in maize, Plant Science, 199 (2013) 103-112.

[28] L.L. Gao, J.P. Anderson, J.P. Klingler, R.M. Nair, O.R. Edwards, K.B. Singh, Involvement of the octadecanoid pathway in bluegreen aphid resistance in Medicago truncatula, Molecular Plant-Microbe Interactions, 20 (2007) 82-93.

[29] C.H. Foyer, G. Noctor, Redox regulation in photosynthetic organisms: signaling, acclimation, and practical implications, Antioxid Redox Sign, 11 (2009) 861-905.

[30] E. Oztetik, A tale of plant glutathione S-transferases: since 1970, Bot Rev, 74 (2008) 419437.

[31] L. Almagro, L.V.G. Ros, S. Belchi-Navarro, R. Bru, A.R. Barcelo, M.A. Pedreno, Class III peroxidases in plant defence reactions, J Exp Bot, 60 (2009) 377-390.

[32] P.Y. Hao, C.X. Liu, Y.Y. Wang, R.Z. Chen, M. Tang, B. Du, L.L. Zhu, G. He, Herbivoreinduced callose deposition on the sieve plates of rice: an important mechanism for host resistance, Plant Physiol, 146 (2008) 1810-1820.

[33] C.M. Smith, E.V. Boyko, The molecular bases of plant resistance and defense responses to aphid feeding: current status, Entomol Exp Applicata, 122 (2007) 1-16.

[34] I. Kaloshian, Gene-for-gene disease resistance: Bridging insect pest and pathogen defense, Journal of Chemical Ecology, 30 (2004) 2419-2438.

[35] G.C. Yencho, M.B. Cohen, P.F. Byrne, Applications of tagging and mapping insect resistance loci in plants, Annu Rev Entomol, 45 (2000) 393-422.

[36] P. Azhaguvel, J.C. Rudd, Y.Q. Ma, M.C. Luo, Y.Q. Weng, Fine genetic mapping of greenbug aphid-resistance gene Gb3 in Aegilops tauschii, Theor. Appl. Genet., 124 (2012) 555564. 
[37] M.D. Lazar, W.D. Worrall, K.B. Porter, N.A. Tuleen, Registration of eight closely related wheat germplasm lines differing in biotype E greenbug resistance, Crop Sci., 36 (1996) 14191419.

[38] Y. Weng, M.D. Lazar, Amplified fragment length polymorphism- and simple sequence repeat-based molecular tagging and mapping of greenbug resistance gene $G b 3$ in wheat, Plant Breed., 121 (2002) 218-223.

[39] M.D. Lazar, G.L. Peterson, J. Hu, Multigenic inheritance of biotype-E greenbug resistance in wheat, Plant Breed., 114 (1995) 492-496.

[40] G.J. Michels, M.D. Lazar, D.A. Fritts, J.D. Booker, Biotype E greenbug reproduction and development through three generations on resistant and susceptible water wheat genotypes, Southw. Entomol., 22 (1997) 431-437.

[41] D.A. Fritts, G.J. Michels, M.D. Lazar, Greenbug dispersal and colonization on a resistant winter wheat genotype: antixenosis, antibiosis or both?, Southw. Entomol., 25 (2000) 113-121. [42] Y. Weng, M.D. Lazar, G.J. Michels, J.C. Rudd, Phenotypic mechanisms of host resistance against greenbug (Homoptera : Aphididae) revealed by near isoaenic lines of wheat, J. Econ. Entomol., 97 (2004) 654-660.

[43] Y.Q. Weng, G.J. Michels, M.D. Lazar, J.C. Rudd, Spatial and temporal distribution of induced resistance to greenbug (Homoptera : Aphididae) herbivory in preconditioned resistant and susceptible near isogenic plants of wheat, J. Econ. Entomol., 98 (2005) 1024-1031.

[44] M.D. Lazar, W.D. Worrall, G.L. Peterson, K.B. Porter, L.W. Rooney, N.A. Tuleen, D.S. Marshall, M.E. McDaniel, L.R. Nelson, Registration of 'TAM 110' wheat, Crop Sci., 37 (1997) 1978-1979.

[45] L. Gautier, L. Cope, B.M. Bolstad, R.A. Irizarry, Affy - analysis of affymetrix GeneChip data at the probe level, Bioinformatics, 20 (2004) 307-315.

[46] R.A. Irizarry, B. Hobbs, F. Collin, Y.D. Beazer-Barclay, K.J. Antonellis, U. Scherf, T.P. Speed, Exploration, normalization, and summaries of high density oligonucleotide array probe level data, Biostatistics, 4 (2003) 249-264.

[47] O. Thimm, O. Blasing, Y. Gibon, A. Nagel, S. Meyer, P. Kruger, J. Selbig, L.A. Muller, S.Y. Rhee, M. Stitt, MAPMAN: a user-driven tool to display genomics data sets onto diagrams of metabolic pathways and other biological processes, Plant J, 37 (2004) 914-939.

[48] B. Usadel, F. Poree, A. Nagel, M. Lohse, A. Czedik-Eysenberg, M. Stitt, A guide to using MapMan to visualize and compare omics data in plants: a case study in the crop species, maize, Plant Cell Environ, 32 (2009) 1211-1229.

[49] A. Rotter, B. Usadel, S. Baebler, M. Stitt, K. Gruden, Adaptation of the MapMan ontology to biotic stress responses: application in solanaceous species, Plant Methods, 3 (2007).

[50] K.J. Livak, T.D. Schmittgen, Analysis of relative gene expression data using real-time quantitative PCR and the $2^{\Delta \Delta} \mathrm{C}(\mathrm{T})$ Method, Methods, 25 (2001) 402-408.

[51] G.A. Howe, G. Jander, Plant immunity to insect herbivores, in: Annu Rev Plant Biol, 2008, pp. 41-66.

[52] M. Wrzaczek, M. Brosche, J. Salojarvi, S. Kangasjarvi, N. Idanheimo, S. Mersmann, S. Robatzek, S. Karpinski, B. Karpinska, J. Kangasjarvi, Transcriptional regulation of the CRK/DUF26 group of receptor-like protein kinases by ozone and plant hormones in Arabidopsis, BMC Plant Biol, 10 (2010).

[53] R.K. Genger, G.I. Jurkowski, J.M. McDowell, H. Lu, H.W. Jung, J.T. Greenberg, A.F. Bent, Signaling pathways that regulate the enhanced disease resistance of Arabidopsis "Defense, No Death" mutants, Mol Plant-Microbe Interact, 21 (2008) 1285-1296. 
[54] M. Seo, T. Koshiba, Complex regulation of ABA biosynthesis in plants, Trends Plant Sci, 7 (2002) 41-48.

[55] K.L.C. Wang, H. Li, J.R. Ecker, Ethylene biosynthesis and signaling networks, Plant Cell, 14 (2002) S131-S151.

[56] Y.P. Qiu, D.Q. Yu, Over-expression of the stress-induced OsWRKY45 enhances disease resistance and drought tolerance in Arabidopsis, Environ Exp Bot, 65 (2009) 35-47.

[57] J. Mizoi, K. Shinozaki, K. Yamaguchi-Shinozaki, AP2/ERF family transcription factors in plant abiotic stress responses, Biochim Biophys Acta, 1819 (2012) 86-96.

[58] A. Schaller, A. Stintzi, Enzymes in jasmonate biosynthesis - structure, function, regulation, Phytochemistry, 70 (2009) 1532-1538.

[59] C. Wasternack, Jasmonates: an update on biosynthesis, signal transduction and action in plant stress response, growth and development, Ann Bot, 100 (2007) 681-697.

[60] J.G. Turner, C. Ellis, A. Devoto, The jasmonate signal pathway, Plant Cell, 14 (2002) S153S164.

[61] A. Stintzi, J. Browse, The Arabidopsis male-sterile mutant, opr3, lacks the 12oxophytodienoic acid reductase required for jasmonate synthesis, Proc Natl Acad Sci USA, 97 (2000) 10625-10630.

[62] A. Stintzi, H. Weber, P. Reymond, J. Browse, E.E. Farmer, Plant defense in the absence of jasmonic acid: the role of cyclopentenones, Proc Natl Acad Sci USA, 98 (2001) 12837-12842.

[63] P.M. Sanders, P.Y. Lee, C. Biesgen, J.D. Boone, T.P. Beals, E.W. Weiler, R.B. Goldberg, The Arabidopsis DELAYED DEHISCENCE1 gene encodes an enzyme in the jasmonic acid synthesis pathway, Plant Cell, 12 (2000) 1041-1061.

[64] S. Blechert, C. Bockelmann, M. Fusslein, T. Von Schrader, B. Stelmach, U. Niesel, E.W. Weiler, Structure-activity analyses reveal the existence of two separate groups of active octadecanoids in elicitation of the tendril-coiling response of Bryonia dioica Jacq, Planta, 207 (1999) 470-479.

[65] C.M. Smith, X.M. Liu, L.J. Wang, X. Liu, M.S. Chen, S. Starkey, J.F. Bai, Aphid feeding activates expression of a transcriptome of oxylipin-based defense signals in wheat involved in resistance to herbivory, J Cheml Ecol, 36 (2010) 260-276.

[66] G. Noctor, A. Mhamdi, S. Chaouch, Y. Han, J. Neukermans, B. Marquez-Garcia, G. Queval, C.H. Foyer, Glutathione in plants: an integrated overview, Plant Cell Environ, 35 (2012) 454-484.

[67] U. Wagner, R. Edwards, D.P. Dixon, F. Mauch, Probing the diversity of the arabidopsis glutathione S-transferase gene family, Plant Mol Biol, 49 (2002) 515-532.

[68] D.P. Dixon, R. Edwards, Glutathione transferases, in: The Arabidopsis Book, The American Society of Plant Biologists, 2010, pp. 1-15.

[69] A. Bresell, R. Weinander, G. Lundqvist, H. Raza, M. Shimoji, T.H. Sun, L. Balk, R. Wiklund, J. Eriksson, C. Jansson, B. Persson, P.J. Jakobsson, R. Morgenstern, Bioinformatic and enzymatic characterization of the MAPEG superfamily, FEBS J, 272 (2005) 1688-1703.

[70] K. Yoshida, P. Kaothien, T. Matsui, A. Kawaoka, A. Shinmyo, Molecular biology and application of plant peroxidase genes, Appl Microbiol Biotech, 60 (2003) 665-670.

[71] C.H. Foyer, G. Noctor, Ascorbate and glutathione: the heart of the redox hub, Plant Physiol, 155 (2011) 2-18.

[72] M. Leterrier, F.J. Corpas, J.B. Barroso, L.M. Sandalio, L.A. del Rio, Peroxisomal monodehydroascorbate reductase. Genomic clone characterization and functional analysis under environmental stress conditions, Plant Physiol, 138 (2005) 2111-2123. 
[73] L. Gilbert, M. Alhagdow, A. Nunes-Nesi, B. Quemener, F. Guillon, B. Bouchet, M. Faurobert, B. Gouble, D. Page, V. Garcia, J. Petit, R. Stevens, M. Causse, A.R. Fernie, M. Lahaye, C. Rothan, P. Baldet, GDP-d-mannose 3,5-epimerase (GME) plays a key role at the intersection of ascorbate and non-cellulosic cell-wall biosynthesis in tomato, Plant J, 60 (2009) 499-508.

[74] A. Wachter, S. Wolf, H. Steininger, J. Bogs, T. Rausch, Differential targeting of GSH1 and GSH2 is achieved by multiple transcription initiation: implications for the compartmentation of glutathione biosynthesis in the Brassicaceae, Plant J, 41 (2005) 15-30.

[75] A. Kessler, I.T. Baldwin, Plant responses to insect herbivory: the emerging molecular analysis, Annu Rev Plant Biol, 53 (2002) 299-328.

[76] D.J. Kliebenstein, Secondary metabolites and plant/environment interactions: a view through Arabidopsis thaliana tinged glasses, Plant Cell Environ, 27 (2004) 675-684.

[77] J. Schwachtje, I.T. Baldwin, Why does herbivore attack reconfigure primary metabolism?, Plant Physiol, 146 (2008) 845-851.

[78] X. Liu, J. Bai, L. Huang, L. Zhu, N. Weng, J.C. Reese, M. Harris, J.J. Stuart, M.S. Chen, Gene expression of different wheat genotypes during attack by virulent and avirulent hessian fly (Mayetiola destructor) larvae, J Chem Ecol, 33 (2007) 2171-2194.

[79] S.J. Park, Y.H. Huang, P. Ayoubi, Identification of expression profiles of sorghum genes in response to greenbug phloem-feeding using cDNA subtraction and microarray analysis, Planta, 223 (2006) 932-947.

[80] F. Divol, F. Vilaine, S. Thibivilliers, J. Amselem, J.C. Palauqui, C. Kusiak, S. Dinant, Systemic response to aphid infestation by Myzus persicae in the phloem of Apium graveolens, Plant Mol Biol, 57 (2005) 517-540.

[81] R.A. Salzman, J.A. Brady, S.A. Finlayson, C.D. Buchanan, E.J. Summer, F. Sun, P.E. Klein, R.R. Klein, L.H. Pratt, M.M. Cordonnier-Pratt, J.E. Mullet, Transcriptional profiling of sorghum induced by methyl jasmonate, salicylic acid, and aminocyclopropane carboxylic acid reveals cooperative regulation and novel gene responses, Plant Physiol, 138 (2005) 352-368. [82] D.R. Bergey, M. Orozco-Cardenas, D.S. de Moura, C.A. Ryan, A wound- and systemininducible polygalacturonase in tomato leaves, Proc Natl Acad Sci USA, 96 (1999) 1756-1760.

\section{Figure Legends}

Fig. 1. MapMan overview analysis identifying functional BINs with respective gene numbers. Differentially expressed transcripts with significant interaction effects (692 transcripts) were used for the functional classification using MapMan. The legend presents the MapMan defined parent BIN number, BIN name, and respective transcripts numbers in the parenthesis; the numbers of transcripts classified under each parent BIN are also presented as data labels.

Fig. 2. Transcripts identified in MapMan biotic stress overview analysis at 0 (A), 24 (B), and 48 (C) hours after greenbug infestation in resistant bulk (RB) compared with susceptible bulk (SB). The $\log 2$ fold changes in the transcript levels were used for the analysis and the color scheme on the scale indicates the nature of gene expression; where blue means "overexpression in RB" and 
red means "overexpression in SB". Each individual box represents a transcript classified under respective functional categories (BINs) defined by the MapMan biotic stress pathway.

Fig. 3. Summary of MapMan biotic stress pathway functional categories that are putatively associated to mediate greenbug feeding induced responses. TF - transcription factors; PR pathogenesis related; GST - glutathione S-transferases.

Fig. 4. Transcripts putatively associated with signaling (A) and phytohormones (B) that are differentially expressed in response to greenbug infestation as defined in MapMan biotic stress pathway. Transcripts with $>2$ fold change (FC) expression difference between resistant bulk (RB) vs. susceptible bulk (SB) at 0, 24, and 48 hai (hours after infestation) are illustrated along with homology based gene names. The symbol "*" indicate the transcript level altered 41 and 93 fold at 24 and 48 hai comparisons respectively and "\#” indicates the transcript with 81 FC at 48 hai. $\mathrm{TF}-$ transcription factors.

Fig. 5. Illustrating transcripts putatively associated to mediate JA biosynthetic pathway in response to greenbug feeding. Each box identifies a single gene differentially expressed between resistant bulk (RB) vs. susceptible bulk (SB) at $0(\mathrm{~A}), 24(\mathrm{~B})$, and 48 (C) hours after infestation (hai). The color scheme on the scale indicates the nature of gene expression presented in $\log 2$ fold change; where blue means "overexpression in RB" and red means "overexpression in SB". $L O X$ - lipoxygenase; 13-HPOT - hydroperoxyoctadecatrienoic acid; 12,13-EOT - 12,13(S)epoxy-octadecatrienoic acid; OPDA -12-oxophytodienoic acid; OPR-OPDA reductase; OPC8:0 - 3-oxo-2-(2'(Z)-pentenyl)-cyclopentane-1-octanoic acid. The illustration is adopted and modified from [59].

Fig. 6. Transcripts putatively associated with glutathione S-transferases (A), peroxidases (B), redox state (C), secondary metabolites (D), and cell wall modifications (E) that are differentially expressed in response to greenbug feeding. Transcripts with $>2$ fold change (FC) expression difference between resistant bulk (RB) vs. susceptible bulk (SB) at 0, 24, and 48 hours after infestation (hai) are presented along with homology based gene names. The symbol “*” adjacent to transcript indicate the expression level in RB vs. SB altered at 21 and 19 fold under 24 and 48 hai; "\#” indicate 13 FC at 48 hai; "\$” indicate 15 and 33 FC at 24 and 48 hai; "^” indicate 28 and 82 FC at 24 and 48 hai; and "@”, indicate 7 and 11 FC at 24 and 48 hai, respectively. 
Fig. 7. qRT-PCR validation of representative transcripts from microarray analysis. The graphs present the magnitude of the differences between resistant (RB) and susceptible (SB) bulks derived from microarray (black filled bars) and qRT-PCR (open bars) for 12 genes. The panel A, C, and E represent the differentially regulated transcripts ( $<10$ fold) between RB vs. SB comparisons at 0, 24, and 48 hours after infestation (hai), respectively. Similarly, panel B, D, and $\mathrm{F}$ present the genes with $>90$ fold change (FC) under the above mentioned treatment comparisons. The two reference genes ('ta.27713.1.a1_x_at' and 'ta.27712.3.a1_x_at') are presented in A, C, and E; and are labeled with a flower bracket.

\section{Supplementary data}

Presents all the differentially regulated transcripts from interaction group (sheet 1), transcripts putatively associated biotic stress by MapMan (sheet 2), and primer combinations used in qRTPCR analysis (sheet 3). 
Fig. 1.

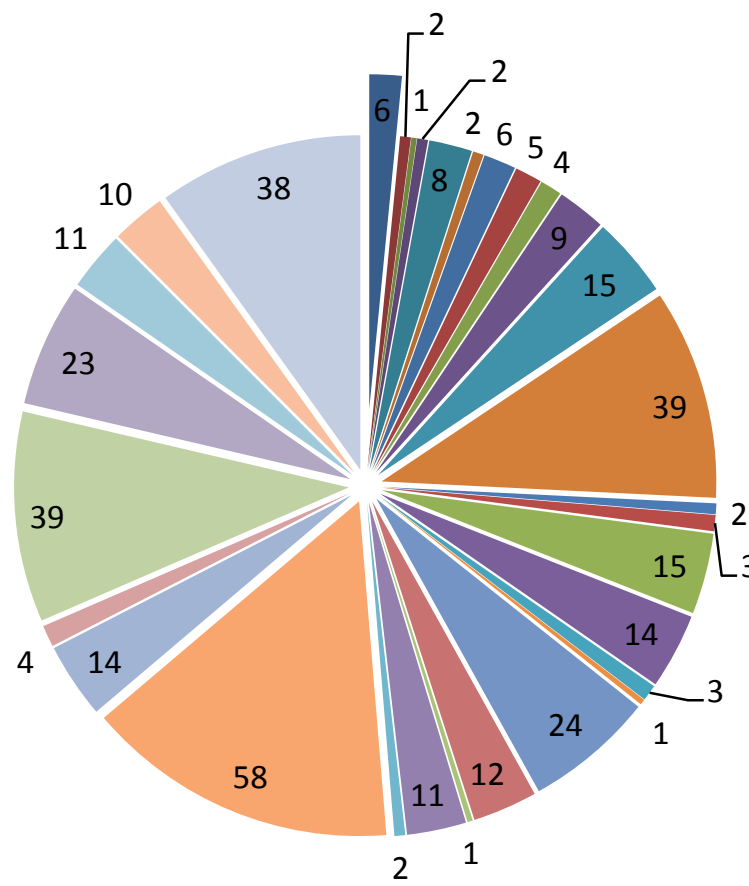

-1. Photosynthesis (6)

- 2. Major $\mathrm{CHO}$ metabolism (2)

- 3. Minor CHO metabolism (1)

4. Glycolysis (2)

5. Fermentation (8)

6. Gluconeogenesis (2)

7. OPP cycle (6)

8. TCA (5)

9. Mitochondrial electron transport (4)

10. Cell wall (9)

11. Lipid metabolism (15)

13. Amino acid metabolism (39)

14. S-assimilation (2)

15. Metal handling (3)

2 16. Secondary metabolism (15)

17. Hormone metabolism (14)

18. Co-factor and Vitamine metabolism (3)

19. Tetrapyrrole synthesis (1)

20. Stress (24)

21. Redox regulation (12)

22. Polyamine metabolism (1)

23. Nucleotide metabolism (11)

25. C1-metabolism (2)

26. Miscellaneous (58)

27. RNA (14)

28. DNA (4)

29. Protein (39)

30. Signalling (23)

31. Cell (11)

33. Development (10)

34. Transport (38)

Fig. 1. MapMan overview analysis identifying functional BINs with respective gene numbers. Differentially expressed transcripts with significant interaction effects (692 transcripts) were used for the functional classification using MapMan. The legend presents the MapMan defined parent BIN number, BIN name, and respective transcripts numbers in the parenthesis; the numbers of transcripts classified under each parent BIN are also presented as data labels. 
Fig. 2.

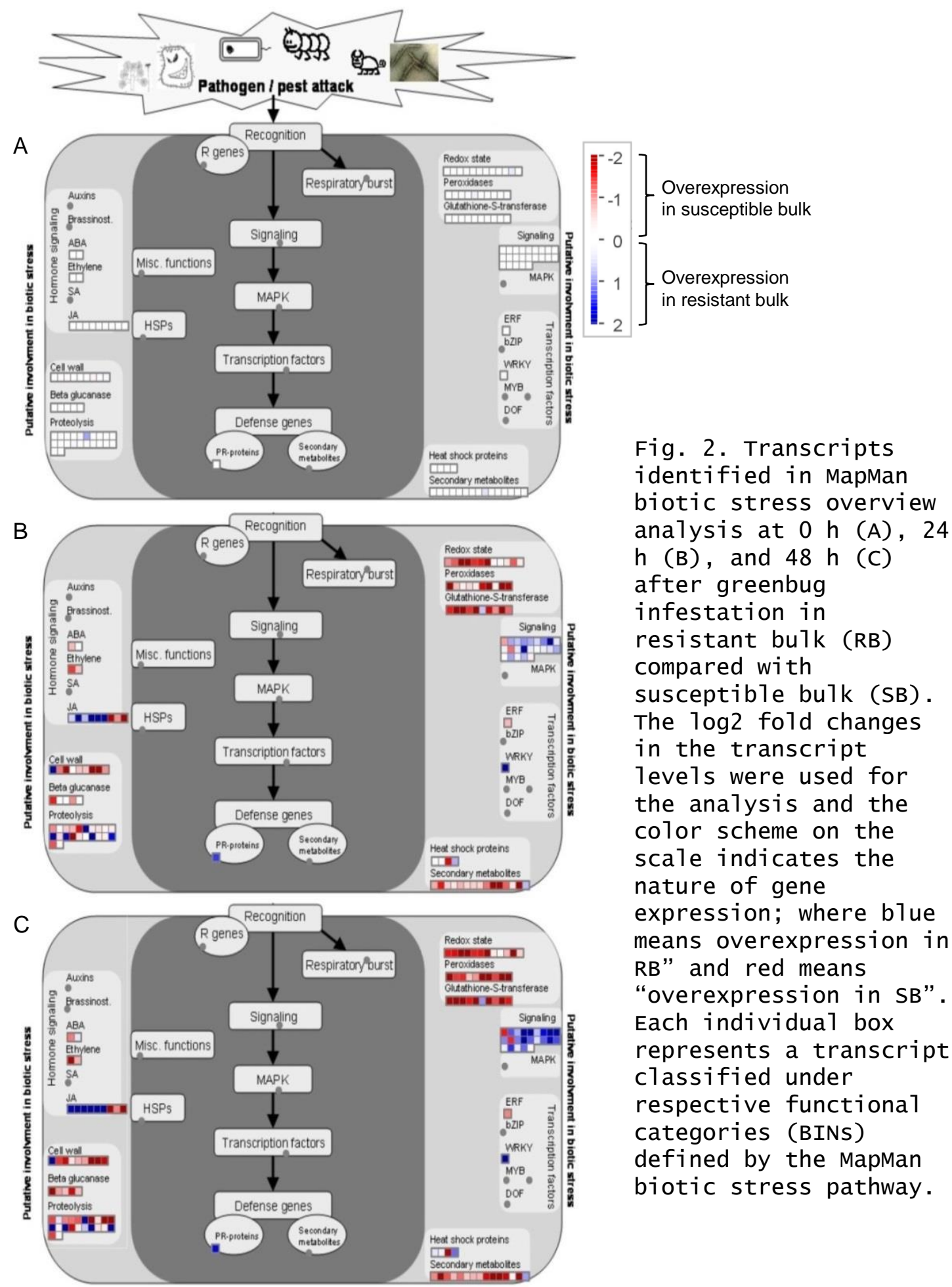


Fig. 3.

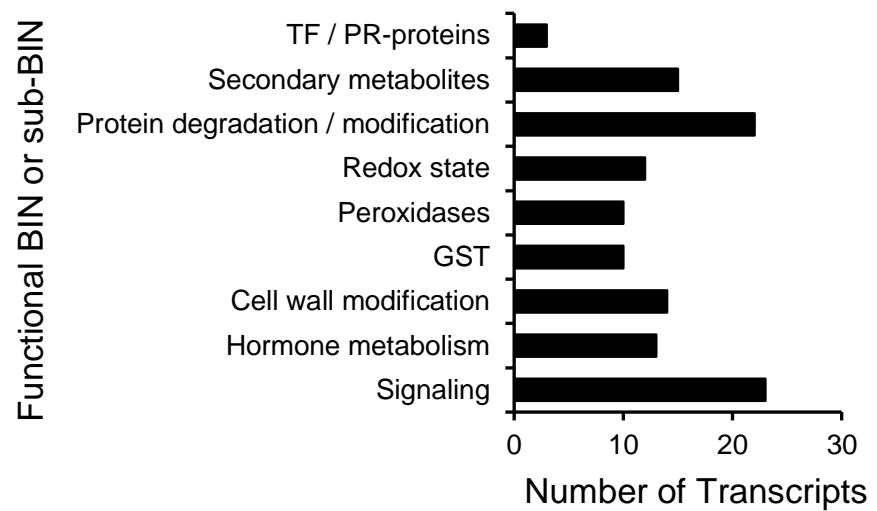

Fig. 3. Summary of MapMan biotic stress pathway functional categories that are putatively associated to mediate greenbug feeding induced responses. TF, transcription factors; PR, pathogenesis related; GST, glutathione s-transferases. (For interpretation of the references to color in this figure legend, the reader is referred to the web version of the article.) 
Fig. 4.

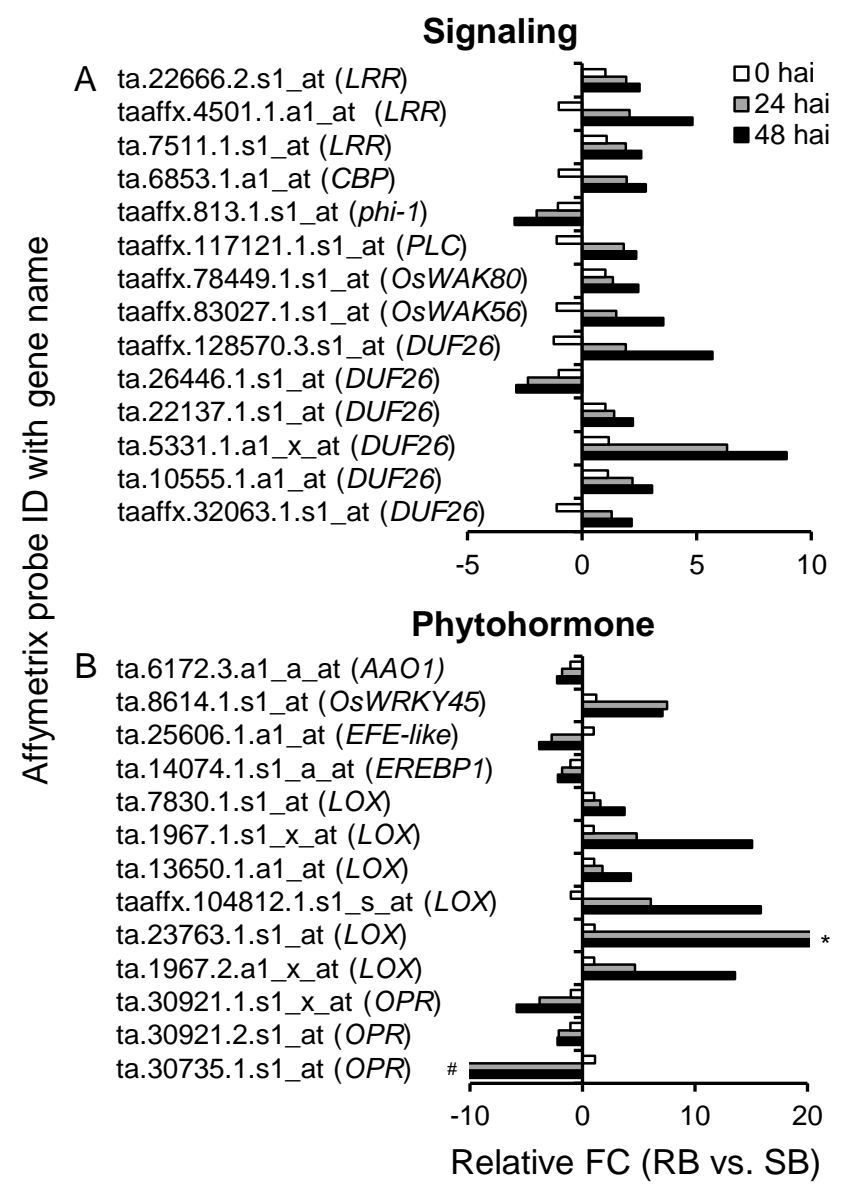

Fig. 4. Transcripts putatively associated with signaling (A) and phytohormones (B) that are differentially expressed in response to greenbug infestation as defined in MapMan biotic stress pathway. Transcripts with $>2$ fold change (FC) expression difference between resistant bulk (RB) vs. susceptible bulk (SB) at 0 hai, 24 hai, and 48 hai (hours after infestation) are i1lustrated along with homology based gene names. The symbol " : $:$ " indicate the transcript level altered 41 and 93 fold at 24 hai and 48 hai comparisons respectively and "\#" indicates the transcript with 81 FC at 48 hai. TF - transcription factors. 
Fig. 5.

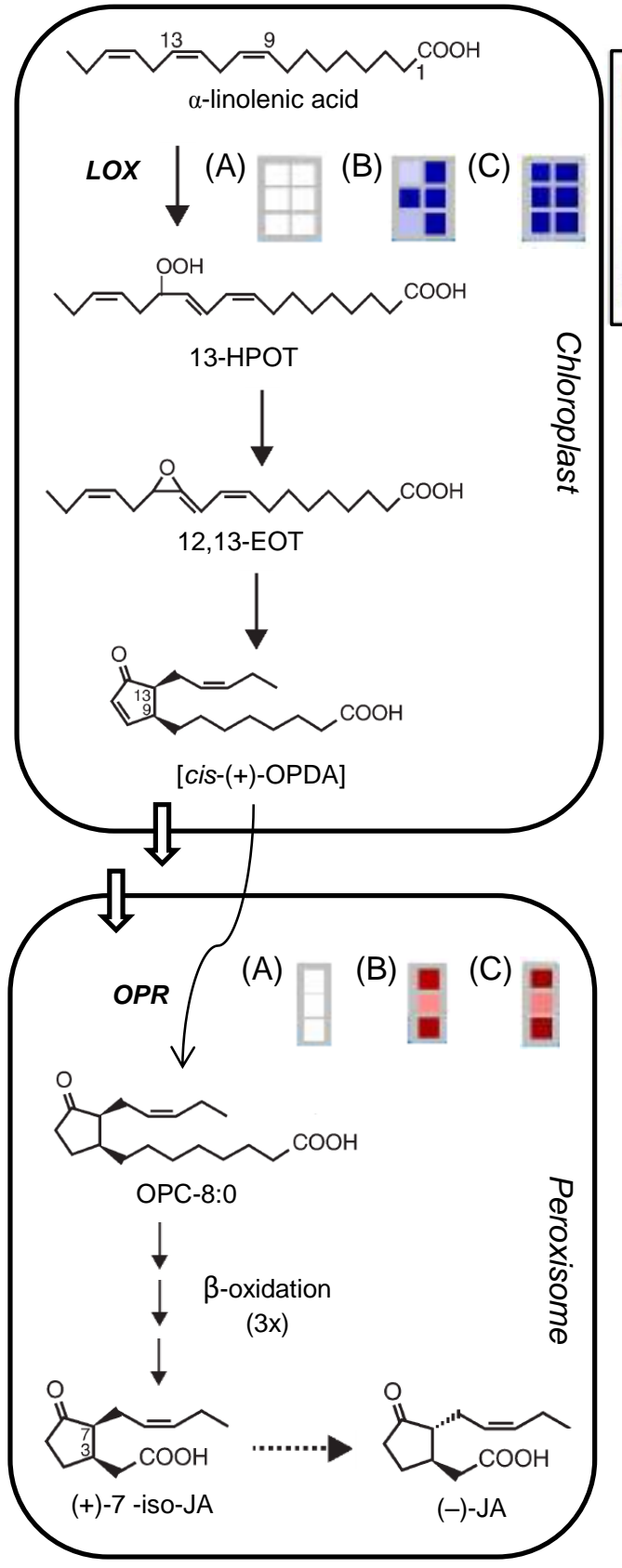

Fig. 5. I7lustrating transcripts putatively associated to mediate JA biosynthetic pathway in response to greenbug feeding. Each box identifies a single gene differentially expressed between resistant bulk (RB) vs. susceptible bulk (SB) at $0 \mathrm{~h}$ (A), $24 \mathrm{~h}$ (B), and $48 \mathrm{~h}(\mathrm{C})$ after infestation (hai). The color scheme on the scale indicates the nature of gene expression presented in 1 og2 fold change; where blue means "overexpression in RB" and red means "overexpression in SB". LOX, 1ipoxygenase; 13-HPOT, hydroperoxyoctadecatrienoic acid; 12,13-EOT, 2,13(S)epoxy-octadecatrienoic acid; OPDA, 12oxophytodienoic acid; OPR, OPDA reductase; OPC-8:0, 3oxo-2-(2_(Z)-penteny 1$)$ cyclopentane-1-octanoic acid. The illustration is adopted and modified from [59]. 
Fig. 6.

\section{Glutathione S-transferases}

A ta.5673.1.s1_at (GST) ta.303.3.s1 at (GSTF2) ta.303.1.s1_at (GSTF2) ta.3679.1.s1_x_at (GSTU6) ta.1878.3.s1_at (GST) taaffx.109595.1.s1_at (GSTF2) ta.303.2.s1_x_at (GSTF2)

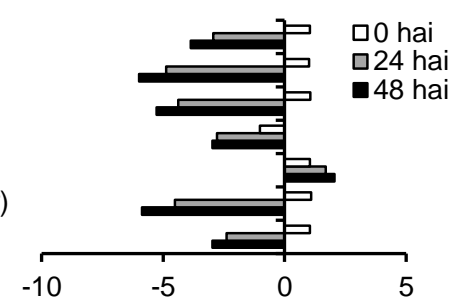

B ta.21307.1.s1_x_at (PER12) ta.21505.1.s1_at (PER4) ta.23376.1.s1_at (PER12) ta.18560.1.s1_s_at (PER2) ta.29496.1.s1_x_at (PER12) ta.23366.3.a1_at (PER2) taaffx.113819.1.s1_at (PER12) ta.21307.2.s1_x_at (PER12)

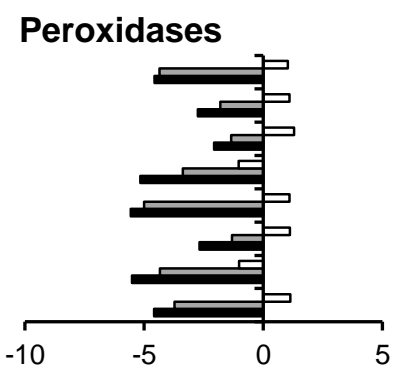

C ta.2706.1.s1_a at $(M D A R)$ ta.3094.1.s1_x_at (GME1) ta.3094.2.s1_at (GME1) ta.5307.1.s1_at (GSH2) taaffx.59101.1.s1_s_at (GSH2) ta.5610.1.s1_at (AHB1) taaffx.25966.1.s1_x_at (CATA2)

Redox homeostasis

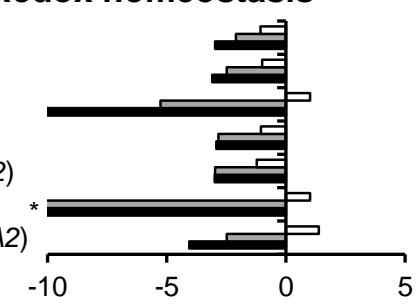

D ta.6247.1.a1_x_at (ACAT2) ta.2925.1.s1_s_at (HMGS) taaffx.86828.1.s1 $\mathrm{x}$ at (HMGS) ta.9592.1.s1_at (FPS1) ta.1465.1.s1_at (PAL1) taaffx.108005.1.s1 at (OMT1) ta.27284.1.s1_at (TDC) ta.2907.1.s1_at (MSDH) taaffx.132143.1.s1_s_at (CYM) ta.5657.1.s1_x_at (OsCCR1)

Secondary metabolites

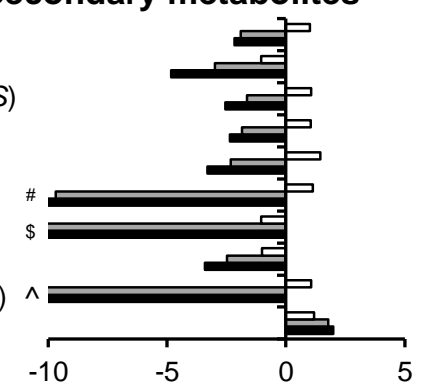

Cell wall modification

E ta.24427.1.s1_at ( $\beta$-1,3-glucanase) ta.3828.3.a1_x_at $(\beta-1,3$-glucanase $)$ ta.5662.1.s1_x_at ( $\beta$-1,3-glucanase) taaffx.107485.1.s1_at (OsCSLA1) ta.7564.1.a1_at (OsCsIE2) ta.4084.1.s1_at (OsCsIE1) ta.25514.1.s1_at (pectinases) ta.8782.1.s1_at (pectinases) ta.8782.2.s1_x_at (pectinases)

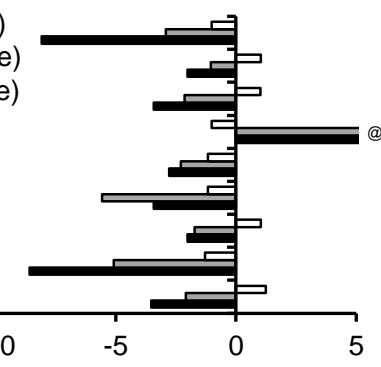

Relative FC (RB vs. SB)
Fig. 6. Transcripts putatively associated with glutathione Stransferases (A), peroxidases (B), redox state (C), secondary metabolites (D), and cell wall modifications (E) that are differentially expressed in response to greenbug feeding. Transcripts with $>2$ fold change (FC) expression difference between resistant bulk (RB) vs. susceptible bulk (SB) at $0 \mathrm{~h}$, $24 \mathrm{~h}$, and $48 \mathrm{~h}$ after infestation (hai) are presented along with homology based gene names. The symbol "*”" adjacent to transcript indicate the expression level in RB VS. SB altered at 21 and 19 fold under 24 hai and 48 hai; "\#” indicate 13 FC at 48 hai; “\$” indicate 15 and 33 FC at 24 and 48 hai; “^' indicate 28 and 82 FC at 24 hai and 48 hai; and "Q" indicate 7 and 11 FC at 24 hai and 48 hai, respectively. 
Fig. 7.
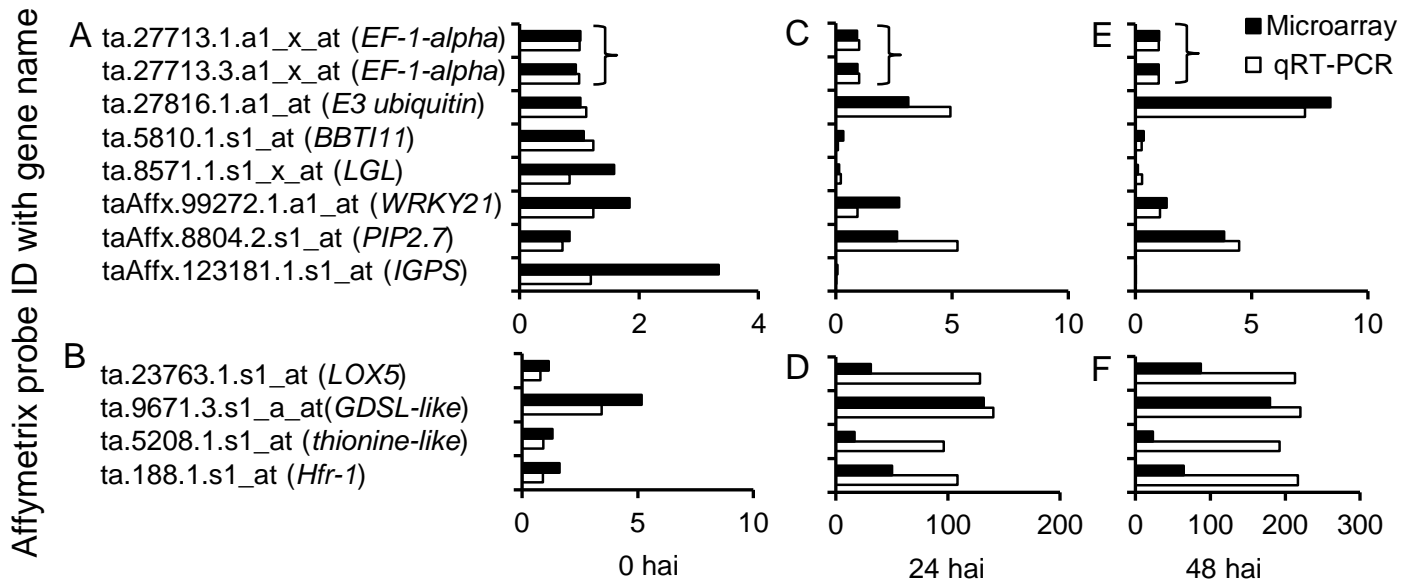

Relative FC (RB vs. SB)

Fig. 7. QRT-PCR validation of representative transcripts from microarray analysis. The graphs present the magnitude of the differences between resistant (RB) and susceptible (SB) bulks derived from microarray (black filled bars) and qRT-PCR (open bars) for 12 genes. The pane $1 \mathrm{~A}, \mathrm{C}$, and $\mathrm{E}$ represent the differentially regulated transcripts $(<10$ fold) between RB vs. SB comparisons at 0,24 , and 48 hours after infestation (hai), respectively. Similarly, panel $B, D$, and $F$ present the genes with $>90$ fold change (FC) under the above mentioned treatment comparisons. The two reference genes ('ta.27713.1.a1 $\mathrm{x}$ at' and 'ta.27712.3.a1 $x$ at') are presented in A, C, and E; and are labeled with a flower bracket. 